\section{A technique for measuring eye-foot coordination without visual guidance*}

\author{
H. H. MIKAELIAN
}

Connecticut College, New London, Connecticut 06320

A device for studying adaptation to rearranged eye-foot coordination is described. The technique allows measurement of changes in the localization of a target with the foot without visual guidance of the responding limb.

Perceptual alterations produced by prolonged viewing through prisms or other optical distorting devices are well documented. The most common design paradigm in these experiments calls for measurements of localizations of a target, either by egocentric orientation or by pointing with a limb, before and then after exposure to the distortion; the magnitude and direction of the differences in localization between pre- and postdistortion experience are a direct measure of the induced perceptual alterations. The apparatus shown in Fig. 1 is designed to study localization of a visual target with the foot (eye-foot coordination) without visual guidance of the responding limb -a device useful in measuring changes in eye-foot coordination that may be produced by looking through prism spectacles. It is constructed to allow $S$ to mark with his foot the location of the virtual image of a target viewed in a mirror. Since view of the marking limb is obstructed by the mirror, guidance of the limb is not contaminated by visual corrective feedback. The design of the present apparatus is based on a tchnique for measuring eye-hand coordination without visual guidance first described by $O . H$. Mowrer in 1935 and later adapted for prism studies by Held and Gottlieb (1958).

The eye-foot coordination test apparatus consists of a truncated rectangular box with a square cross section ( $39 \times 26 \times 14$ in.). One end of the box is fitted with a first-surface mirror at $45 \mathrm{deg}$ to the longitudinal axis, while the opposite end contains the target lights. A set of goggles is mounted on the top cover above the mirror so that $S$, resting his head on the goggles, can view the reflection of the targets in the mirror. The box cover is also hinged at the target end to allow access to the target lights. The entire apparatus is mounted on a wall at a height of 39 in. A $S$ viewing through the goggles perceives the virtual image of the target cast on the floor and marks its location on a sheet of paper with a stylus attached to his shoe. We mounted the stylus on the sawed-off anterior portion of a skate with toe clamps (see insert) which could be easily fitted to S's shoe. Different-shaped styli are used to differentiate markings for the different conditions.

The four targets, mounted in an irregular quadrilateral shape on the back surface of the box, consist of pilot lights whose intensity can be controlled with a variable resistor. A rotary switch is used to present each target singly and in a predetermined random order; in the present apparatus, one full rotation of the switch was designed to display each of the four targets four times. Target intensity is adjusted to make it clearly visible without illuminating any other part of the box (the box is painted flat black inside to reduce reflection from its walls).

Sample data from a prism rearrangement experiment are presented in Table 1 to illustrate variability of responses as well as the magnitude of adaptation that was measured by this apparatus. Exposure to rearrangement entailed moving one leg while viewing it through 20 diopter wedge prisms that displaced the visual field laterally. The prism goggles were mounted over an opening on a slanted table top so that $\mathbf{S}$ could rest his head on the goggles while viewing his leg movements. This method diminishes fatigue substantially, while allowing $S$ to hold his head relatively immobile during exposure.

The specific procedure of the experiment was as follows: After preexposure localizations of the four targets (using a pointed-tip stylus), S viewed his leg movements for $50 \mathrm{~min}$, following which he again marked the location of the targets (using a stylus with a circular cutting tip). Both sets of markings were done on the same sheet of paper. Differences between pre- and postexposure markings for each target were calculated as follows: The horizontal distance of each preexposure mark from a convenient reference line was measured; the mean of these measurements represented the horizontal centroid of the markings

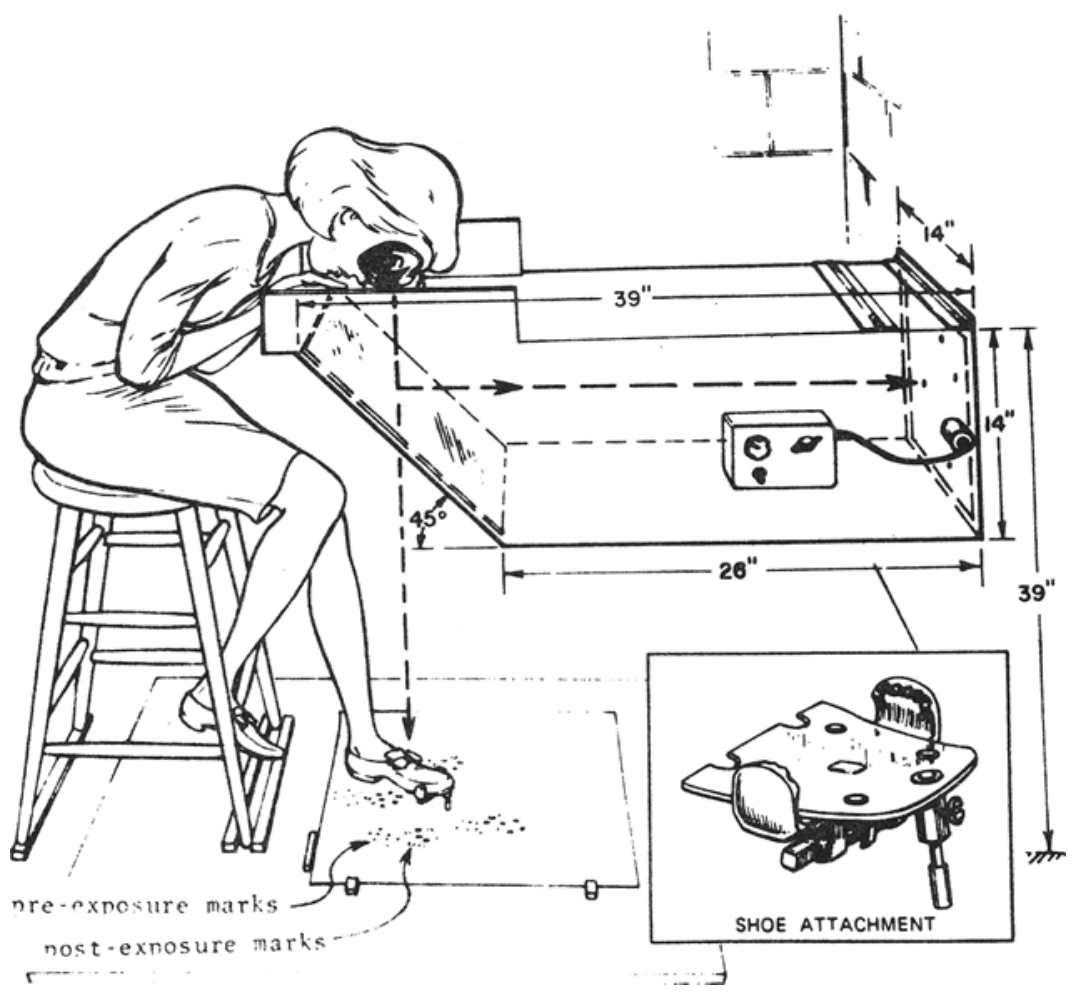

*This study was supported by N.I.H. Grant MH 11801-01.
Fig. 1. Eye-foot coordination test apparatus. 
Table 1

Changes in Eye-Foot Coordination Following $50 \mathrm{Min}$ of Viewing Leg Movements Through 20-Diopter Wedge Prisms (in Inches)

\begin{tabular}{|c|c|c|c|c|c|}
\hline \multicolumn{3}{|c|}{ Base Left } & \multicolumn{3}{|c|}{ Base $R$ ight } \\
\hline & \multicolumn{2}{|c|}{ Exposed Limb } & \multirow[b]{2}{*}{$\mathbf{S}$} & \multicolumn{2}{|c|}{ Exposed Limb } \\
\hline $\mathbf{S}$ & $\begin{array}{l}\text { Left } \\
\text { Foot }\end{array}$ & $\begin{array}{c}\text { Right } \\
\text { Foot }\end{array}$ & & $\begin{array}{l}\text { Left } \\
\text { Foot }\end{array}$ & $\begin{array}{c}\text { Right } \\
\text { Foot }\end{array}$ \\
\hline 1 & 2.2 & 0.7 & 7 & 1.0 & 1.3 \\
\hline 2 & 4.4 & 6.4 & 8 & 0.7 & 1.4 \\
\hline $\mathbf{3}$ & 3.4 & 4.8 & 9 & 2.2 & 1.5 \\
\hline 4 & 2.3 & 4,0 & 10 & 1.2 & 2.3 \\
\hline 5 & 3.1 & 2.6 & 11 & 1.5 & 0.7 \\
\hline 6 & 4.1 & 3.0 & 12 & 0.9 & 1.0 \\
\hline Mean & 3.25 & 3.6 & Mean & 1.25 & 1.36 \\
\hline p* & 0.001 & 0.01 & $\mathbf{p}^{*}$ & 0.01 & 0.01 \\
\hline
\end{tabular}

*t test

for that target. The centroid for postexposure markings of that target, using the same reference line, was similarly calculated. The magnitude and the direction of the difference between the centroids of the pre- and postexposure markings represented the perceived lateral displacement of that target. This procedure was repeated for all four targets, and the mean displacement of the four targets for each exposure condition is reported in Table 1. Since pre- and postexposure measurements were taken without prisms, the displacements represent aftereffects of prism exposure.

The data are reported in terms of linear displacement of target localizations, although they can be readily converted into degrees of visual angle (in the present apparatus, a mean linear displacement of 1 in. is equivalent to an angular displacement of $1.8 \mathrm{deg}$ ). The measurements reported in Table 1 are quite stable, with variability no greater than that observed in measurements of eye-hand coordination (Held \& Hein, 1958). Since this method of measuring eye-foot response is analogous to that of eye-hand coordination, direct comparison of the data between the two types of responses is made feasible.

\section{REFERENCES}

HELD, R., \& GOTTLIEB, N. Technique for studying adaptations to disarranged eye-hand coordinations. Perceptual \& Motor Skills, 1958, 8, 83-86.

HELD, R., \& HEIN, A. Adaptation of disarranged hand-eye coordination contingent upon re-afferent stimulation. Perceptual \& Motor Skills, 1958, 8, 87-90.

MOWRER, O. H. A device for studying eye-hand coordination without visual guidance. American Journal of Psychology, 1935, 47, 493-495. 